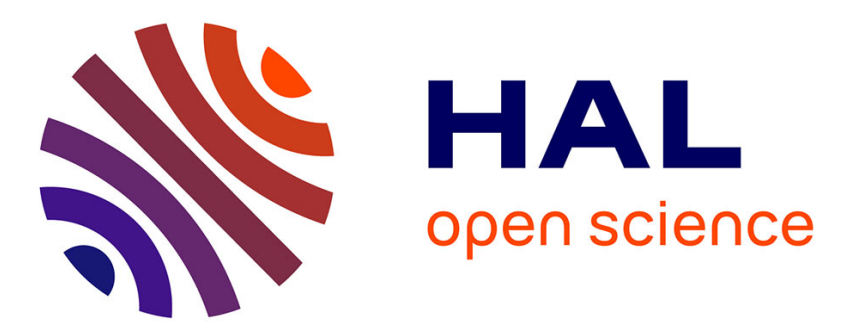

\title{
3D numerical simulation of the behaviour of a spherical particle suspended in a Newtonian fluid and submitted to a simple shear
}

\author{
Jérôme Bikard, Pierre Menard, Edith Peuvrel-Disdier, Tatiana Budtova
}

\section{- To cite this version:}

Jérôme Bikard, Pierre Menard, Edith Peuvrel-Disdier, Tatiana Budtova. 3D numerical simulation of the behaviour of a spherical particle suspended in a Newtonian fluid and submitted to a simple shear. Computational Materials Science, 2006, 37 (4), pp.Pages 517-525. 10.1016/j.commatsci.2005.12.003 . hal-00574190

HAL Id: hal-00574190

https://hal-mines-paristech.archives-ouvertes.fr/hal-00574190

Submitted on 25 Jul 2012

HAL is a multi-disciplinary open access archive for the deposit and dissemination of scientific research documents, whether they are published or not. The documents may come from teaching and research institutions in France or abroad, or from public or private research centers.
L'archive ouverte pluridisciplinaire HAL, est destinée au dépôt et à la diffusion de documents scientifiques de niveau recherche, publiés ou non, émanant des établissements d'enseignement et de recherche français ou étrangers, des laboratoires publics ou privés. 


\title{
3D numerical simulation of the behaviour of a spherical particle suspended in a Newtonian fluid and submitted to a simple shear
}

\author{
Jérôme BIKARD*, Pierre MENARD, \\ Edith PEUVREL-DISDIER, Tatiana BUDTOVA
}

\author{
Ecole Nationale Supérieure des Mines de Paris, \\ Centre de Mise en Forme des Matériaux, UMR CNRS / Ecole des Mines 7635, BP 207, \\ 06904 Sophia-Antipolis France
}

\begin{abstract}
The 3D flow around a rigid spherical particle suspended in a Newtonian fluid and submitted to simple shear is numerically studied using Rem $3 \mathrm{D}^{\circledR}$ finite element code. The sphere motion is imposed by a sticking contact between the sphere and the fluid. The effect of the particle size as compared with the finite dimension of the shear cell was investigated. The direct calculations show that $3 \mathrm{D}$ modelling is necessary to correctly predict the sphere behaviour. The proximity of the particle and the cell walls strongly affects the flow velocities, the sphere motion (increase of the rotation period of the sphere) and the stress field (change of orientation angle and increase of maximal local stresses).
\end{abstract}

Keywords: hydrodynamic stresses, suspended sphere, size effect, 3D flow, finite element method, multidomain approach

\footnotetext{
* To whom the correspondence should be addressed e-mail: Jerome.Bikard@ensmp.fr, tel: +33 (0)4 939575 55, fax: +33 (0)4 92389752
} 


\section{INTRODUCTION}

The comprehension and the prediction of the behaviour of objects (rigid particle, agglomerated fillers, liquid drop, gel particles or capsules, etc) suspended in a matrix and submitted to a flow require a good knowledge of the mechanical fields (velocity, hydrodynamic stresses) around this object. For example, the dispersion of agglomerated fillers (such as carbon black, silica, calcium carbonate) in a polymer matrix, which is performed in an internal mixer or an extruder, depends on the local hydrodynamic stresses applied on the pellet. For example, dispersion occurs as soon as the hydrodynamic stress overcomes the agglomerates' cohesion [1, 2]. During mixing, the size of the pellet and the flow cell can be of similar dimension. This may affect the local hydrodynamic stresses around the pellet as compared with their distribution in an infinite medium. As a result, the behaviour of the particle will be modified. It is thus important to know the local stresses applied on the pellet and to consider the potential wall effect due to the finite cell dimensions.

The behaviour of objects of basic shapes (spheres) in an infinite Newtonian matrix is well described by analytical solutions in the case of pure shear [3, 4]. The particle rotates with a constant period which is inversely proportional to the shear rate and the theoretical tensile stress around the particle is maximal at $45^{\circ}$ and $225^{\circ}$ [5]. However this analysis does not take into account the finite dimensions of the cell around the object nor their consequences on the sphere behaviour. More complex flows (for example, with several spheres) were considered analytically first by [6] and then completed by [7]. But the effect of the restricted flow field dimension was not explicitly taken into account in their analyses. Recently, [8] used a 3D numerical simulation in order to calculate the effect of the finite dimension of the shear cell on the rotation period of a sphere. The sphere rotation is imposed by considering a condition of zero torque on the sphere surface. The rotation rate of the particle is determined by assuming that the global torque applied on the sphere is also equal to zero. In both cases it was shown that streamlines are influenced by the sphere size and the rotation period differs from the one obtained by Jeffery. No results on the stress or strain fields were reported. 
The objective of this work is to use a finite element method to solve the motion equation for a rigid sphere suspended in a Newtonian matrix and submitted to simple shear and to study the effect of the proximity of the cell walls on the behaviour of the sphere. The Rem $3 \mathrm{D}^{\circledR}$ software is used to calculate the flow and the hydrodynamic stresses around the sphere. A multidomain approach and a sticking condition at the sphere/matrix interface are considered.

The equations of the problem and the numerical method to solve them are described in section 2 . The direct results of the three-dimensional simulation of the flow around the sphere in a finite matrix (impact of the size effect on the streamlines, hydrodynamic stresses and rotation period) are presented in section 3 and compared with the ones known from literature.

\section{THEORETICAL BACKGROUND}

In this study, a numerical simulation is used to calculate the behaviour of a rigid sphere suspended in a Newtonian fluid and submitted to a constant shear rate in a restricted geometry. The Rem $3 \mathrm{D}^{\circledR}$ software initially dedicated to the injection of the polymers $[9,10,11]$ is used to calculate the flow and the hydrodynamic stresses around the rigid sphere. The shear flow is imposed by two planes rotating in the opposite direction. The infinite medium is restricted by the finite gap between the translating planes (see Fig.1). The shear cell is considered as a multiphase domain consisting of the sphere and the Newtonian fluid.

\section{Figure 1}

\subsection{Mechanical equations and boundary conditions}

The rigid sphere is approximated by a "droplet" of a Newtonian fluid with the viscosity $\eta_{\text {part }}$ much higher and density $\rho_{\text {part }}$ being the same than the ones of matrix $\eta_{f}$ and $\rho_{f}$, respectively (e.g. $\eta_{\text {part }} / \eta_{f}>10^{3}$; cf. Table 1 ). The viscosity difference ensures 
rigid body behaviour of the sphere. Equal densities allow neglecting gravity effect. The behaviour of the fluid is assumed to follow a Newtonian law, though viscoelastic cases could be considered (forthcoming study). The contact between the fluid and the sphere (described by the surface $\Sigma$ ) is a perfect sticking. The system can be described by the following set of equations:

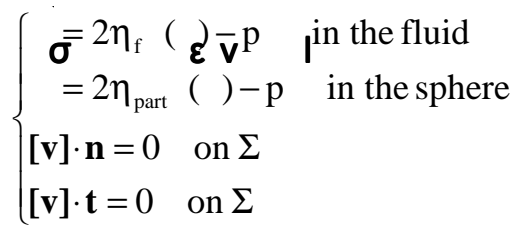

where $\mathbf{v}, \boldsymbol{\varepsilon ( \mathbf { v } )}$ and $p$ are the velocity, the deformation rate and the local pressure, respectively, [v] represents the jump of the velocity field on the particle surface $\Sigma$ and I is the identity tensor.

\section{Table 1}

The schematic presentation of the shear cell with a control volume $\Omega=\mathrm{L} * \mathrm{~h} * \mathrm{e}$, where $\mathrm{L}$ is its length, e is the width and $\mathrm{h}$ is the distance between the upper and lower plates (the gap size) is shown in Fig.1. e and L dimensions are assumed to be equal and $\mathrm{h}$ is much smaller than $\mathrm{L}$ which reflects experimental conditions [12]). For the sake of symmetry, the sphere is located in the middle of the gap. The boundary conditions on the border $\partial \Omega$ are given by the following relations:

$$
\left\{\begin{array}{ll}
\mathrm{p}_{1}=\mathrm{p}_{2} & \text { on } \partial \Omega_{\mathrm{p}} \\
\mathrm{v}_{1}=-\mathrm{v}_{2} & \text { on } \partial \Omega_{\mathrm{v}}
\end{array} \text { where } \partial \Omega_{\mathrm{p}} \cup \partial \Omega_{\mathrm{v}}=\partial \Omega ; \partial \Omega_{\mathrm{p}} \cap \partial \Omega_{\mathrm{v}}=\phi\right.
$$

The cell dimensions relatively to the sphere size were chosen in such a way that the "non disturbed" kinematics of the fluid far from the sphere corresponds to pure shear. Inertial effects are neglected, thus the non disturbed flow is quasi twodimensional (in the (E,G) plane). A zero constant pressure is imposed on the vertical walls (i.e. $p_{1}=p_{2}=0$ ), and thus the calculated pressure will directly correspond to the perturbation caused by the presence of a rotating sphere. 


\subsection{Numerical considerations}

In order to solve equations (1) and the evolution of the surface $\Sigma$ as a function of time, a characteristic function $\mathbf{C}_{f}$ is defined as a new field in the control volume $\Omega$ :

$$
\mathbf{C}_{f}(x, t)= \begin{cases}1 & \text { if } x \text { is in fluid } \\ 0 & \text { if } x \text { is in the particle }\end{cases}
$$

where $x$ represents the Cartesian coordinates of a material point in the control volume $\Omega$.

The local balance equations in the sphere and in the fluid can be written in the volume $\Omega$ with the virtual work principle as follows:

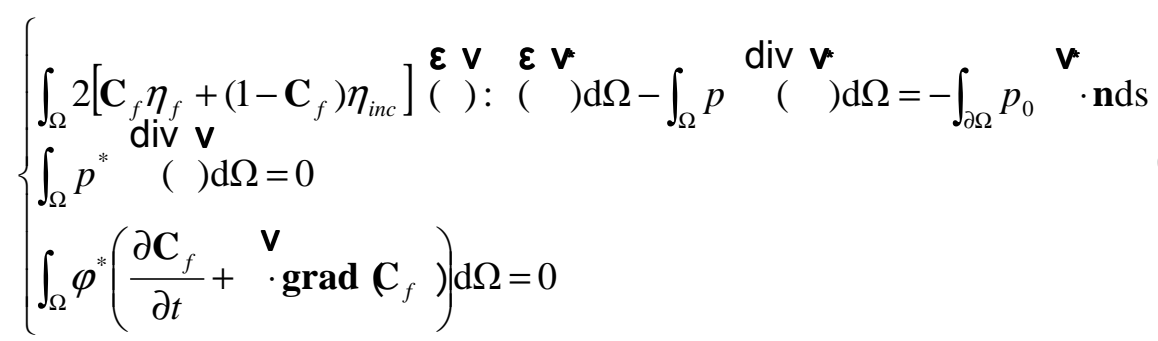

where $\left({ }^{\mathbf{v}^{*}}, p^{*}, \varphi^{*}\right)$ are functions chosen in adapted Sobolev $[9,10]$. The first equation in the set (4) expresses the mechanical quasi-static balance, the second - the incompressibility of fluids and the third - the convection of the characteristic function. This last equation means that the only one allowed degree of freedom for the sphere is a rotation around the axis $\mathrm{V}$ (in agreement with the boundary conditions). The surface conditions are trivially satisfied in these equations and thus the formulation of the problem is completed.

The interface $\Sigma$ between the sphere and the fluid mathematically corresponds to the surface of discontinuity of the characteristic function $\mathbf{C}_{f}$ through the elements of mesh [13]. In terms of distributions, the interface can be defined by:

$$
\sum=\left\{x \in \Omega / \operatorname{grad}\left(\mathbf{C}_{f}(x)\right)=\delta_{x} \mathbf{n}\right\}
$$

where $\delta_{x}$ is the Dirac distribution on $x$. 
The equations (4) are discretised by the discontinuous Galerkin method with mixed pressure/velocity finite elements (see e.g. $[9,10,13,14,15])$. The choice of a non structured mesh with tetrahedral elements with a bubble stabilisation ensures the numerical stability of the method (with respect to the Ladyszhenskaya, Babuska and Brezzi criterion $[16,17]$. The mesh of the control volume $\Omega$ is based on adaptive techniques of anisotropic meshes and detection of interfaces [11] and uses the $\mathrm{GCM}^{\circledR}$ software associated to Rem $3 \mathrm{D}^{\circledR}$.

As shown in Fig.2, the use of adaptive mesh and algorithms is essential for a correct location of the interface fluid/sphere. Fig.2a shows the initial interface between the sphere and the fluid and Fig.2b - a large numerical diffused interface due to a too coarse mesh. Fig.2c shows an adapted mesh around the interface and Fig.2d - the excellent calculation of the characteristic function. The mesh adaptation is correlated to the detection of the interface. This technique enables to minimize the numerical error on the location of the interface. Figure 3 shows a cross section of the meshed volume with the anisotropic adapted mesh.

\section{Figure 2}

\section{Figure 3}

\section{RESULTS AND DISCUSSION}

The following dimensions of the control volume are considered: $\mathrm{e}=\mathrm{L}=20 \mathrm{~mm}$ and $\mathrm{h}=0.1 \mathrm{~mm}$. The velocity of the walls is equal to $0.25 \mathrm{~mm} \cdot \mathrm{s}^{-1}$ leading to a macroscopic shear rate of $5 \mathrm{~s}^{-1}$. The applied shear rate is fixed constant in the numerical simulation. The other parameters are given in Table 1 . The Reynolds number for the flow is equal to $R_{e}=1.210^{-7}$ which means that the flow is purely laminar. The dimension of the sphere (diameter $=d$ ) as compared with the distance between the translating walls $($ gap $=h$ ) is described by the ratio: 


$$
r=\frac{d}{h}
$$

All simulations are performed in 3D. A sensibility analysis towards the variation of particle viscosity was performed. It showed that the results do not depend on the viscosity of the particle if the ratio (particle viscosity)/(fluid viscosity) is greater than $10^{3}$. The calculated streamlines, stress fields and resulting rotation periods are described in the following sections.

\subsection{Velocity fields and streamlines around the sphere}

Figure 4 shows the calculated velocity fields around the sphere in the (E,G) median plane which contains the equator of the sphere for several (sphere diameter)/(gap size) ratios. The results exhibit a recirculation zone with strong shearing. The streamlines that are in this recirculation zone make a half-turn before reaching the sphere and the local flow direction (in the vicinity of the sphere) becomes opposite to the direction of the main flow. This zone of high shear rate corresponds to a zone of high shear stress and thus of viscous dissipation. The presence of a singularity point in such a flow was also predicted by [7].

\section{Figure 4}

The effect of the $r$ ratio on the dimensions of the recirculation zone is depicted in Fig.4a-d for $r=0.2,0.4,0.6$ and 0.8 , respectively. The greater is the $\mathrm{r}$ ratio (the larger is the sphere relatively to the gap size and thus the closer to the sphere are the walls), the larger is the recirculation zone. Moreover, the recirculation zone moves closer to the sphere with $r$ increase. As it will be shown in part 3.2, this can be correlated to the normal stress increase and the tangential stress decrease with the growth of $r$ value (sphere diameter becoming comparable to the gap size).

In order to better characterise the importance of this recirculation, the main recirculation zone is described by its thickness $1(\mathrm{x})$ (see Fig.4a), $\mathrm{x}$ being the distance 
from the sphere centre. The variation of this thickness reduced to the sphere diameter $1(\mathrm{x}) / \mathrm{d}$ versus $\mathrm{x} / \mathrm{d}$ reduced distance is plotted on Fig. 5 for different $\mathrm{r}$ ratios. This shows that the maximal size of the main recirculation zone is of the order of the sphere dimension and increases with the ratio $\mathrm{r}$ increase. This can also qualitatively be seen from Fig.4.

\section{Figure 5}

The position of $1(\mathrm{x}) / \mathrm{d}$ local minimum indicates the location of the zone of the highest shear rate due to the opposite flows and thus of high shear stress. For example, for $r=0.2,1(\mathrm{x})=0$ when $\mathrm{x}=\mathrm{d}$ (see Fig.5, curve 1); this means that the zone of high shear stress is located at the distance from sphere centre equal to its diameter. The corresponding area is shown by a circle in Fig.4a. For $r=0.4,1(\mathrm{x})=0$ when $\mathrm{x}<\mathrm{d}$ (see Fig. 5, curve 2): the point of singularity is moving closer to the sphere surface $(x / d<1)$. While the value of the minimum of $1(\mathrm{x})$ is equal to zero for $r=0.2$ and $r=0.4$, this minimum value increases for $r>0.4$. For $r=0.8$, the zone of high stress due to the opposite flows touches the sphere surface $(1(x)$ is minimal for $x<d / 2)$. This means that for $r>0.4$ the zone of high stress is not reduced to a singularity point but becomes larger and larger and encircles part of the sphere (see Fig 4d). A similar behaviour was also predicted by [7] in their analytical solution. As it will be shown in the part 3.2, the presence of high shear rate zone in the vicinity of the sphere can be correlated with the evolution of the angle at which the stress is maximal as the dimension of the sphere becomes comparable to the gap size.

The effect of the third dimension (along the $\mathrm{V}$ axis) on the velocity fields is shown in Figure 6. An example of six cross sections in the (E,G) plane cutting the sphere at different distances from its centre at $\mathrm{V}=0$ (equatorial plane), $\mathrm{d} / 4, \mathrm{~d} / 2, \mathrm{~d}$ (tangential to the sphere surface), $2 \mathrm{~d}$ and $4 \mathrm{~d}$ is shown for the case $r=0.6$. Fig.6a is equivalent to Fig. $4 \mathrm{c}$ but represented in 3D. Figure $6 \mathrm{~b}$ demonstrates that the recirculation area is not confined in the equatorial plane but also has a thickness in the third dimension V. This is confirmed in Figure $6 \mathrm{c}$ where a deflection of velocity lines appears around the sphere (with swirls in $\mathrm{V}$ direction). The streamlines do not remain in the 
cross section plane but curve in the third dimension (warping around the sphere). Figures 6a-c shows that there is a second recirculation area around the sphere (closed streamlines). The closed loops around the sphere correspond to a layer in which an object orbits around the sphere. These closed streamlines exist only in the vicinity of the sphere (see Figure $6 \mathrm{~d}$ and $6 \mathrm{e}$ ). At $\mathrm{V}=2 \mathrm{~d}$ only the main recirculation area exists (no closed streamlines around the sphere), and the warping effect is less pronounced. At V $>3-4 \mathrm{~d}$, streamlines are not disturbed by the presence of the sphere anymore and are parallel (corresponding to a shear field) (Fig.6f).

\section{Figure 6}

Fig. 6 clearly shows the importance of a 3D modelling. The appearance of a second recirculation zone could not be noticed in $2 \mathrm{D}$ calculations, because the flow field is too constrained. This difference between normal and tangential stress values obtained in $2 \mathrm{D}$ and $3 \mathrm{D}$ modelling will be discussed in part 3.2.3.

\subsection{Stress and velocity fields around the sphere}

This part demonstrates the effect of the sphere diameter relatively to the gap size on the normal and tangential stresses and velocity profiles around the sphere. The results concerning the velocity and stress fields around the sphere are presented in Figs. 7 and 8 , respectively.

\subsubsection{Consequence of the proximity of the walls on the velocity field}

Fig. 7 shows the velocity field inside and outside the sphere for $r=0.2$ and $r=$ 0.8 , in an equatorial cross-section in $(\mathrm{E}, \mathrm{G})$ plane. The results are as follows:

- the velocity field inside the sphere (which is approximated by a very viscous fluid) corresponds to the rotation field of a solid;

- in the case of quasi-infinite medium ( $r=0.2$, Fig 7a) the velocity field outside the sphere corresponds to a pure shear (the velocity is proportional to the distance from the sphere). This result correspond to the classical theory;

- in the case of finite medium ( $r=0.8$, Fig $7 \mathrm{~b})$, the velocity field corresponds to a Poiseuille shear (with parabolic velocity profiles). This result can be explained 
by the fact that the proximity of the plates generates a flow around the sphere with a strong pressure gradient (Poiseuille flow) as shown in Fig. 4.

\section{Figure 7}

\subsubsection{Consequence of the proximity of the plates on the stress field}

For normal and tangential stresses a known analytical solution was developed for a sphere in an infinite medium. In this case, normal and tangential stresses are given by Brenner equations [5]:

$$
\left\{\begin{array}{l}
\mathbf{F}_{\mathbf{n}}=\mathbf{n} \cdot \boldsymbol{\sigma} \cdot \mathbf{n}=\frac{5}{2} \eta_{f} \dot{\gamma} \sin (2 \theta) \\
\mathbf{F}_{\mathbf{t}}=\mathbf{t} \cdot \boldsymbol{\sigma} \cdot \mathbf{n}=\frac{5}{2} \eta_{f} \dot{\gamma} \cos (2 \theta)
\end{array}\right.
$$

The theoretical maximal normal stress $\mathbf{F}_{\mathbf{n}}$ is obtained at a $\theta$ angle equal to $45^{\circ}$ (curve 1 in Fig.8a). For a 200 Pa.s viscosity and a $5 \mathrm{~s}^{-1}$ shear rate, the value of the maximal theoretical normal stress is equal to $2.510^{3} \mathrm{~Pa}$.

\section{Figure 8}

Fig. 8 shows the calculated dependence of normal and tangential stresses on angle $\theta$ for different $r$ ratios. Two cases can be clearly distinguished: $r \leq 0.2$ (quasiinfinite medium) and $r>0.2$.

a) $\underline{r \leq 0.2}$

- The maximal normal stress obtained for $r=0.2$ is at $\theta=45.03^{\circ}$ (see Fig.8a), which corresponds to Brenner theory prediction. The difference between the calculated and theoretical values of $\theta$ is less than $0.1 \%$. The value of the calculated maximal normal stress is $2513 \mathrm{~Pa}$ (less than $1 \%$ difference with the value obtained via Brenner equations, which can be explained by the fact that the assumption of quasi-infinite medium is not fully satisfied with $r=0.2$ ). This explains why the curves of the normal stress versus orientation angle for the 
analytical solution and for the numerical simulation at $r=0.2$ are practically coinciding and the case of $r \leq 0.2$ can be considered as a quasi-infinite medium.

- The zero tangential stress obtained for $r=0.2$ is at $\theta=45.02^{\circ}$ (see Fig.8b). The difference between the calculated and the theoretical values is also lower than $0.1 \%$. The variation of the orientation angle leads to the change in the sign of the tangential stress: it is positive in the flow direction $\left(\theta \rightarrow 0^{\circ}\right)$ and negative being perpendicular to the flow $\left(\theta \rightarrow 90^{\circ}\right)$

b) $\underline{r>0.2}$

The analytical prevision in an infinite medium [4] is not valid in this case. The 3D simulation shows that:

- the angle $\theta$ at which the normal stress is maximal is displaced from $45^{\circ}$ to higher values with the $\mathrm{r}$ ratio increase (curves 3-5 in Fig.8a) and the curves become asymmetrical (the angle is moving towards the closest plate). The maximal value of the normal stress is also increased with the r ratio. For example, with $r$ variation from 0.4 to 0.9 the maximal normal stress evolves from about $3000 \mathrm{~Pa}$ to about $30000 \mathrm{~Pa}$, which corresponds to a high super-pressure in the fluid near the sphere;

- the value of $\theta$ at which the tangential stress becomes equal to zero is also shifted from $45^{\circ}$ to $65^{\circ}$ (curves 3-5 in Fig.8b). In other words, for $r$ between 0.4 and 0.9, the angle at which the tangential stress is zero moves towards the direction of the wall and the maximal tangential stress increases from $2500 \mathrm{~Pa}$ to about $13000 \mathrm{~Pa}$. The curves of the tangential stresses become asymmetrical as well as the ones for the normal stresses.

The global net torque applied on the sphere was also calculated. In the case of an infinite medium $(r \leq 0.2$, the torque is equal to zero, as predicted by Brenner (see Eq. (7)). For the case of a finite medium, the calculated torque remains equal to zero, as assumed by [8] in their simulation. 


\subsubsection{Discussion: comparison between the stress field in $2 D$ and $3 D$}

The angle values at which the normal stresses are maximal and the tangential stresses are equal to zero were calculated in 2D and 3D approximation. The comparison is depicted in Fig.9a,b where the difference between numerical and analytical (quasiinfinite medium) stresses calculated 2D and 3D modelling is shown. The main result is that $2 \mathrm{D}$ calculation is overestimating these angles. The difference between $2 \mathrm{D}$ and $3 \mathrm{D}$ simulations is increasing with the $\mathrm{r}$ ratio increase. As shown in Part 3.1, 2D simulation cannot correctly predict all the aspects of the flow around the sphere. The constrained flow in $2 \mathrm{D}$ is at the origin of the overestimated stress values in this geometry. $2 \mathrm{D}$ simulations should not be used to interpret flow fields around spheres in a finite medium.

\section{Figure 9}

The results obtained with Rem $3 \mathrm{D}^{\circledR}$ modelling are shown by curve 2 in Fig.10. In this case, the motion of the sphere occurs due to the sticking condition between the sphere and the suspending fluid.

\section{Figure 10}

The results of both numerical simulations coincide with Jeffery period for $r<$ 0.2 , which is given by the following relation [3]:

$$
T_{\text {Jeffery }}=\frac{4 \pi}{\dot{\gamma}}
$$

The sticking condition predicts a deceleration of sphere rotation as the sphere diameter relatively to the gap size ratio increases. This is in contradiction with the simulation assuming a condition of zero torque (Pomchaitaward et al. model). The recirculation area and the point of singularity are not reported in this work [8]. In the present modelling, the deceleration is explained by the presence of zones of high shear rates (opposite flows in a narrow dimension) corresponding to a zone of strong 
mechanical energy dissipation as identified on Fig 4a-d. This dissipation, increasing with $r$, is correlated to a reduction of the mechanical energy in the flow and thus of kinetic energy resulting in a decrease of velocity. This decrease of velocity in the flow then results in an increase of the rotation period of the sphere. Above $r>0.9$ calculations cannot be performed because of convergence problems when $r$ approaches 1 , even by refining the meshes between the sphere and the plates. However, when the sphere diameter is equal to the gap size $(r=1)$, the period of rotation should be $T=$ $2 \pi / \dot{\gamma}$ according to the sticking contact between the sphere and the walls, which gives half of Jeffery period.

Using a method of least square approximation, the dependence of the rotation period $T$ on $r$ for ratios $r$ in the [0;0.9] interval was determined as follows:

$$
T(r)=T_{\text {Jeffery }}\left(1-2.212 r+0.642 r^{2}\right)
$$

The choice of a parabolic approximation has been made for simplicity reasons, and the coefficients in equation (9) were estimated for a fixed shear rate of $5 \mathrm{~s}^{-1}$. This formula was checked to be valid for a Newtonian liquid for shear rates between $0.5 \mathrm{~s}^{-1}$ and $10 \mathrm{~s}^{-1}$.

The overall results obtained show the importance of considering the proximity of the particle and the wall in the shear cell. The ratio (particle diameter/gap size) has to be taken into account during experiments, especially if willing to have a simple shear and to avoid swirls and fluid recirculation. This problem concerns different types of systems and phenomena, for example, shear-induced rupture of agglomerates [18] or release of solvent from a swollen micro-gel or micro-capsule $[19,20,21]$.

\section{CONCLUSIONS}

The behaviour of a sphere suspended in a Newtonian fluid and submitted to a simple shear was modelled by 3D numerical simulation using the Rem3D ${ }^{\circledR}$ software based on finite elements approximation. The classical Kao, Cox and Mason approach with sticking condition between the sphere and the fluid was used as a basis of the 
modelling. The effect of the sphere diameter relatively to the gap size on the velocity pattern, stress distribution around the sphere, streamlines and period of rotation was considered and compared with results obtained in the framework of other approaches.

For a sphere diameter/gap size ratio lower than 0.2 the numerical results correspond to the analytical solutions of [3] and [5]. For diameter/gap ratios greater than 0.2 , the effects of the proximity of the walls towards the sphere are significant:

- the period of rotation increases as the sphere and the gap become of a comparable size,

- a recirculation zone appears leading to an increase of the maximal normal stresses around the sphere and a shift of the angle where this stress is maximum towards higher values,

- the appearance of swirls of streamlines in the $\mathbf{V}$ axis which shows the importance of the modelling in 3D.

The calculated flow with $\operatorname{Rem} 3 \mathrm{D}^{\circledR}$ showed a good agreement with [7] analytical results (case of a complex shear generated by the proximity of the plates). However, the results obtained are in disagreement with the rotation period of the sphere predicted by [8]. The two simulations differ in their hypothesis to impose the motion of the sphere. In the present case, the evolution of the calculated period has been correlated with the velocity and stress fields and the mechanical dissipation in the flow.

\section{ACKNOWLEDGEMENTS}

Authors are grateful to Veronique Collin (present address: Lafarge, France) and Sylvie Vervoort, former PhD students of CEMEF-Ecole des Mines de Paris, for the stimulating discussions. 


\section{REFERENCES}

[1]. W.R. Bohlen, R.E. Colwell, Intensive mixing, Soc. Plast. Eng. J., 14 (1958) 2428.

[2]. J.M. MacKelvey, Mixing chapt. 12, Polymer Processing, John Wiley and Sons Inc.: New York (1962) 299.

[3]. G.B. Jeffery, The motion of ellipsoidal particles immersed in a viscous fluid, Proc. R. Soc. London, Ser A 102 (1922) 161-179

[4]. D.F. Bagster, D. Tomi, The stresses within a sphere in simple flow fields, Chem. Eng. Sci. 29 (1974) 1773-1783

[5]. H. Brenner, The stockes resistance of an arbitrary particle III. Shear fields, Chem. Eng. Sci. 19 (1964) 631-651

[6]. W. Bartok, S.G. Mason, Particle motions in sheared suspensions. VII Internal circulation in fluid droplets (theorical), J. Coll. Sci. 13 (1958) 293-307

[7]. S.W. Kao, R.G. Cox, S.G. Mason, Streamlines around single spheres and trajectories of pairs of spheres in two dimensional creeping flows, Chem. Eng. Sci. 32 (1977) 1505-1515

[8]. C. Pomchaitaward, I. Manas-Zloczower, D.L. Feke, Investigation of the dispersion of carbon-black agglomerates of various sizes in simple-shear flows, Chem. Eng. Sci. 58 (2003) 1859-1865

[9]. E. Pichelin, T. Coupez, Finite element solution of the 3D mold filling problem for viscous incompressible fluid, Comp. Meth. Appl. Mech. Eng. 163 (1998) 359-371

[10]. E. Pichelin, T. Coupez, A taylor discontinuous Galerkin method for the thermal solution in 3D mold filling, Comp. Meth. Appl. Mech. Eng. 178 (1999) 153-169

[11]. T. Coupez, E. Bigot, 3D anisotropic mesh generation and adaptation with applications, Proc. European congress in Comp. Meth. Appl. Sci. Eng. (2000)

[12]. O. Seyvet, P. Navard, Collision-Induced Dispersion of Agglomerate Suspensions in a shear Flow, J. Appl. Polym. Sci., 78 (2000) 1130-1133.

[13]. C. Béraudo, A. Fortin, T. Coupez, Y. Demay, B. Vergnes, J.F. Agassant, A finite element method for computing the flow of multi-mode viscoelastic fluids: comparison with experiments, J. Non Newtonian Fluid Mech. 75 (1997) 1-23. 
[14]. P. Lesaint, P.A. Raviart, Mathematical aspects of finite elements in partial differential equations. Academic Press (1974).

[15]. G.M. Hulbert, T.J.R. Hughes, Space-time finite element formulation for incompressible viscous flows. Comp. Meth. Appl. Mech. Eng. 84 (1990) 327-348.

[16]. I. Babuska, W.C. Rheinboldt, Adaptive approaches and reliability estimations in finite element analysis, Comp. Meth. Appl. Mech. Eng., 17-18(3) (1979) 519-540

[17]. T.J.R. Hughes, L.P. Franca, G.M. Hulbert, A new finite element formulation for computational fluid dynamics: VIII the Galerkin/least-square method for advective-diffusive equations. Comp. Meth. Appl. Mech. Eng. 73 (1989) 173-179.

[18]. V. Collin, Etude rhéo-optique des mécanismes de dispersion du noir de carbone dans des élastomères, $\mathrm{PhD}$ thesis, Cemef, Ecole des Mines de Paris, SophiaAntipolis (2004)

[19]. A. Zanina, T. Budtova, Hydrogel under shear: a rheo-optical study of the particle deformation and solvent release, Macromolecules, 35 (2002) 1973-1975.

[20]. S. Vervoort, T. Budtova, Evidence of shear-induced polymer release from a swollen gel, Polym. International, 52 (2003) 553-558.

[21]. A. Zanina, A. Vilesov, T. Budtova, Shear-induced solvent release from gel particles: application to drug-delivery systems, Int. J. Pharmaceutics, 242 (2002) 137-146. 


\section{FIGURE CAPTIONS}

\section{Figure 1}

Schematic presentation of the flow cell of volume $\Omega$ (a) and of the particle with the surface $\Sigma(\mathrm{b})$. The trihedral (E, G, V) corresponds to the global referential, whereas (n, t, V) corresponds to the local one.

\section{Figure 2}

Comparison between interface calculations without and with mesh adaptation (a and c), and corresponding numerical diffusion of the characteristic function as a result of the simulation (b and d).

\section{Figure 3}

Cross-section of the volume $\Omega$ in (E,G) plane: anisotropic mesh in a non disturbed area, adapted around the particle.

\section{Figure 4}

Simulated flow around the particle in the cross-section (in (E,G) plane) and position of the area with the highest shear stress for different $r$ values.

\section{Figure 5}

Thickness of the recirculation area $1(\mathrm{x}) / \mathrm{d}$ versus the distance from the centre of the sphere $\mathrm{x} / \mathrm{d}$ for several $\mathrm{r}=\mathrm{d} / \mathrm{h}$ values. 


\section{Figure 6}

3D streamlines in the equatorial (E,G) plane around and in front of the particle for several cross sections.

\section{Figure 7}

A vertical cross-section (in $(\mathrm{E}, \mathrm{G})$ plane) of the flow cell with a rigid particle, showing velocity field for $r=0.2(a)$ and $0.8(b)$.

\section{Figure 8}

Normal (a) and tangential (b) stresses as a function of angle $\theta$ for the infinite medium (1) and several values of ratio $r=0.2(2), 0.6(3), 0.8(4)$ and $0.9(5)$.

\section{Figure 9}

Difference between numerical and theoretical (in a quasi-infinite medium) maximum normal (a) and tangential forces (b) in 2D and 3D.

\section{Figure 10}

Rotation periods as a function of (particle diameter)/(gap size) ratio: Jeffery reference (1), present modelling in Rem $3 \mathrm{D}^{\circledR}(2)$ and zero torque approach [8] (3). 
Table 1. Geometrical and material characteristics used in calculations

\begin{tabular}{|l|l|l|l|}
\hline & Particle diameter or gap size $(\mu \mathrm{m})$ & Viscosity $(\mathrm{Pa} \mathrm{s})$ & Density $\left(\mathrm{kg} / \mathrm{m}^{3}\right)$ \\
\hline Particle & From 20 to 80 & $2.510^{5}$ & $0.9710^{3}$ \\
\hline Fluid & 100 & $210^{2}$ & $0.9710^{3}$ \\
\hline
\end{tabular}




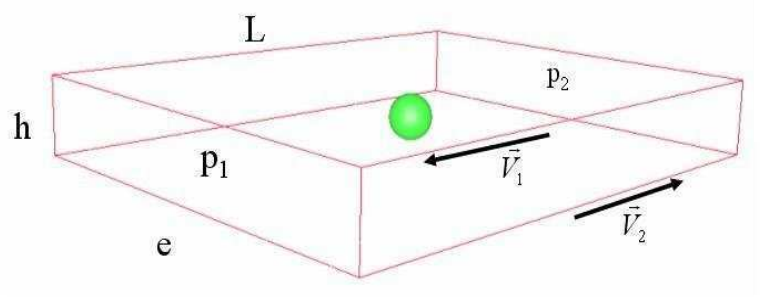

(a)

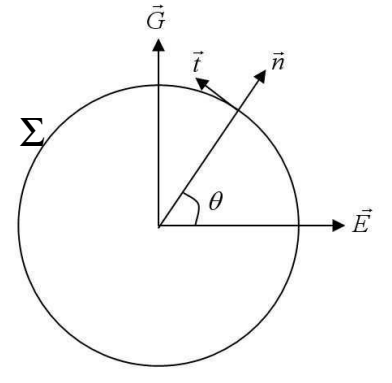

(b)

Figure 1 


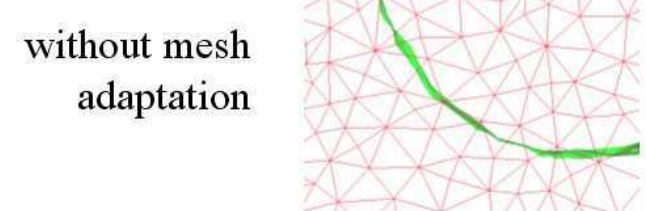

with mesh adaptation (GCM) (a)
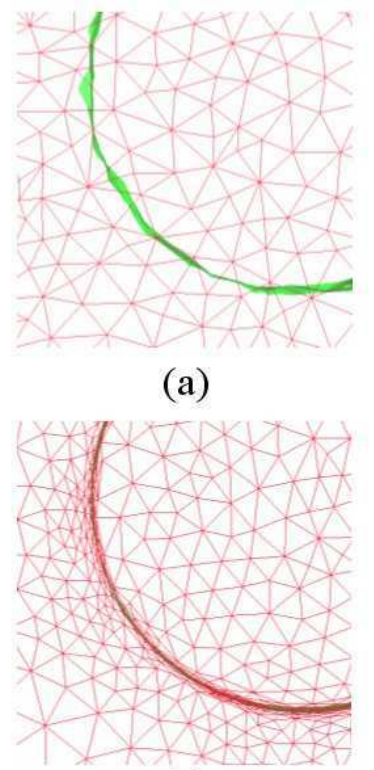

(c)

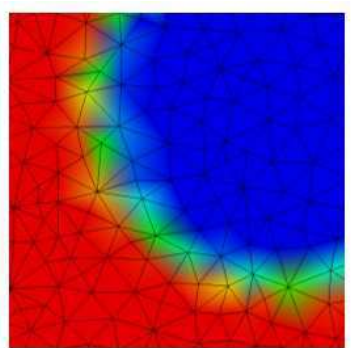

(b)

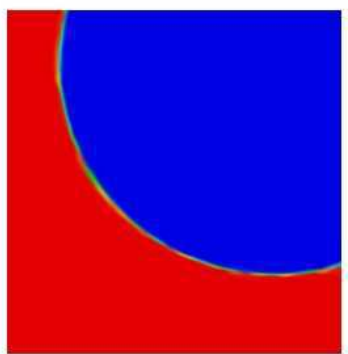

(d)

Figure 2 


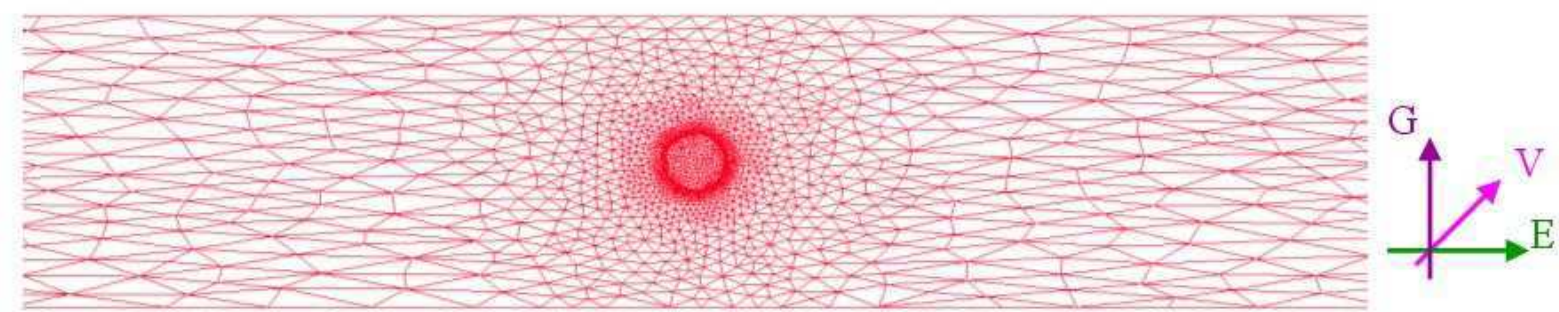

Figure 3 


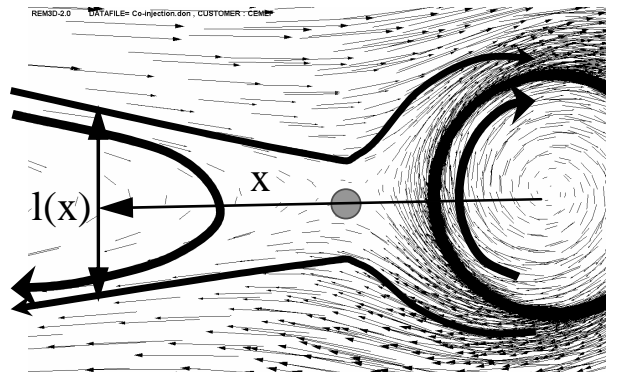

(a) $r=0.2$

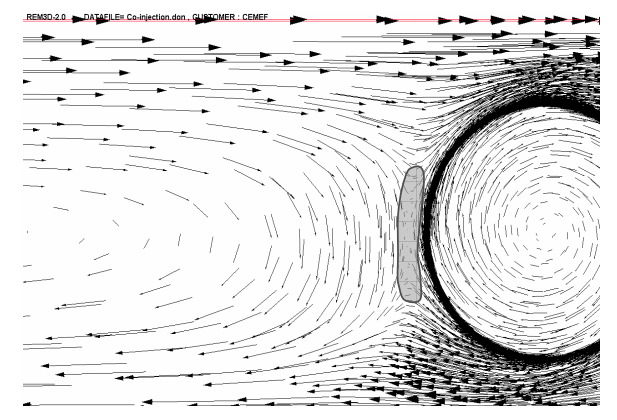

(c) $r=0.6$

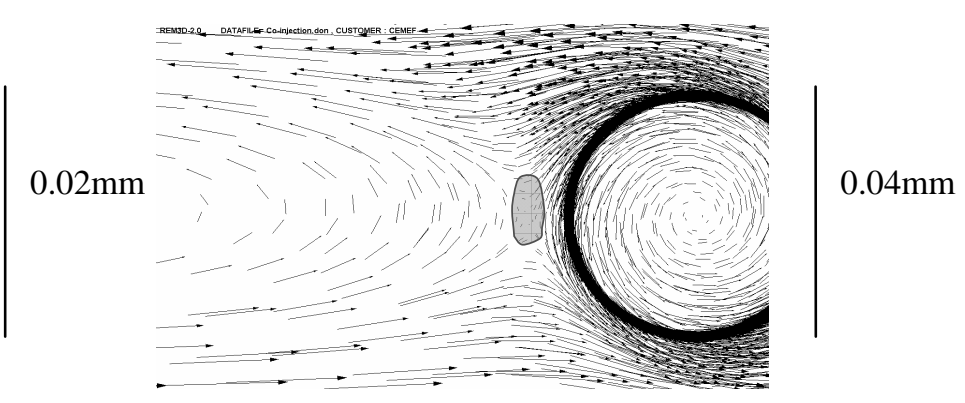

(b) $r=0.4$

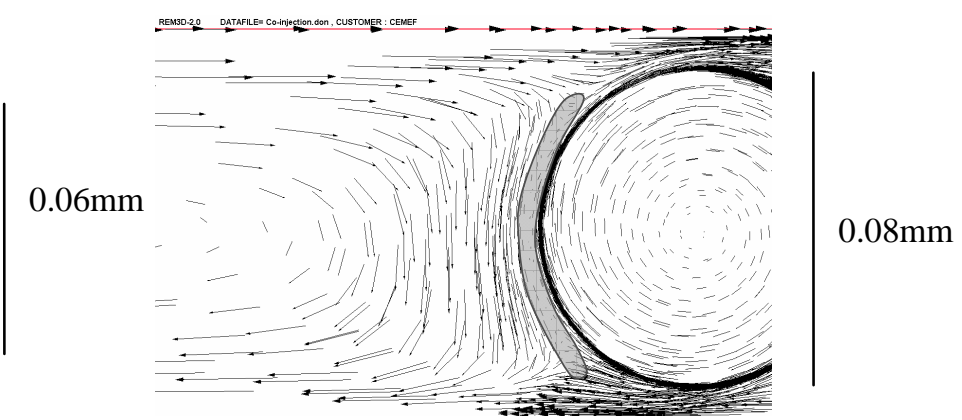

(d) $r=0.8$

Figure 4 


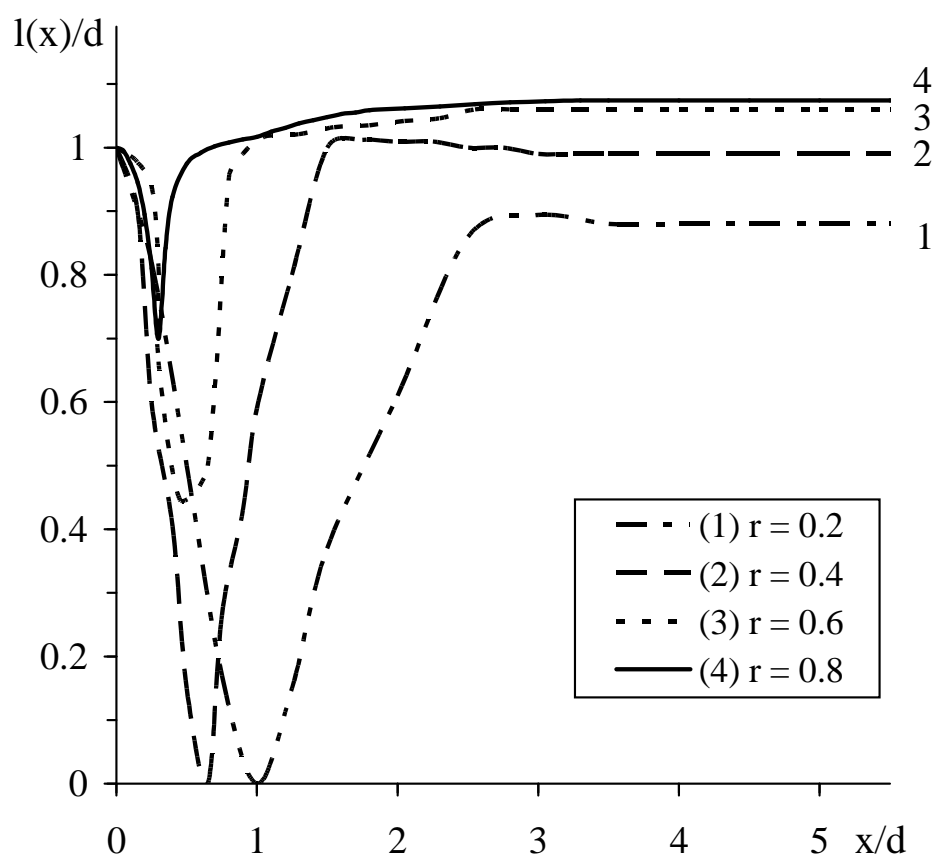

Figure 5 


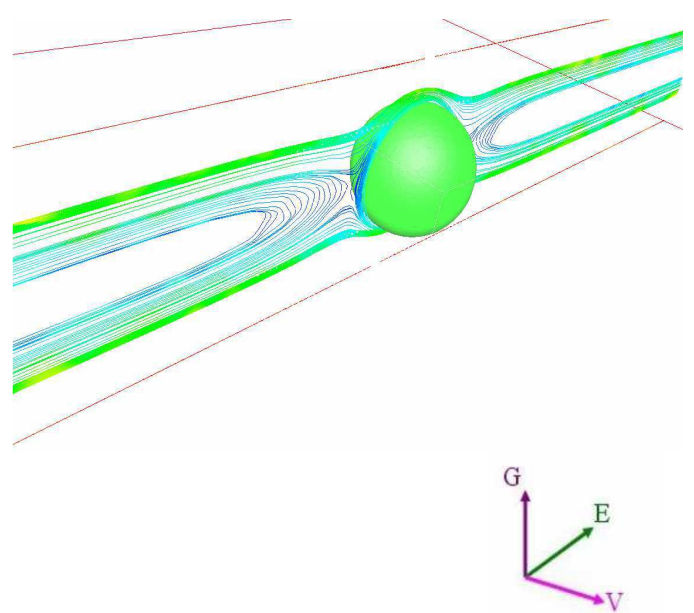

(a) $\mathrm{V}=0$

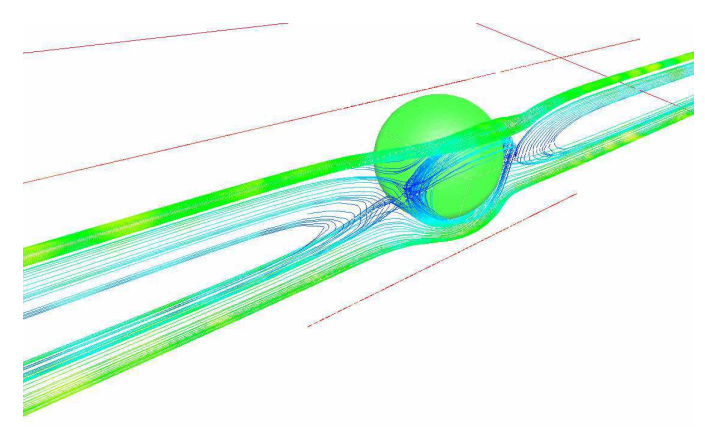

(c) $\mathrm{V}=\mathrm{d} / 2$

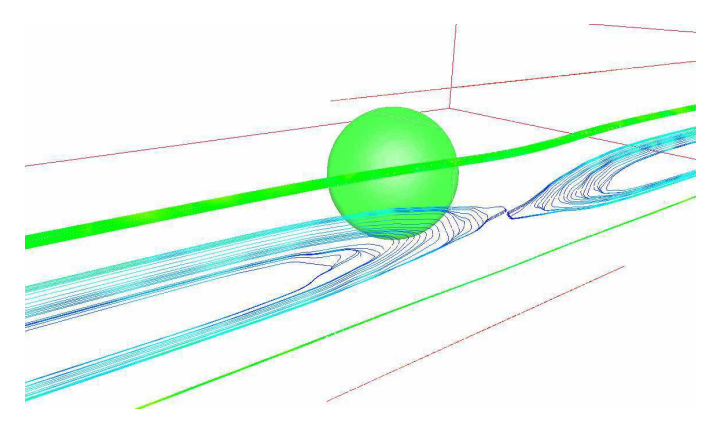

(e) $\mathrm{V}=2 \mathrm{~d}$

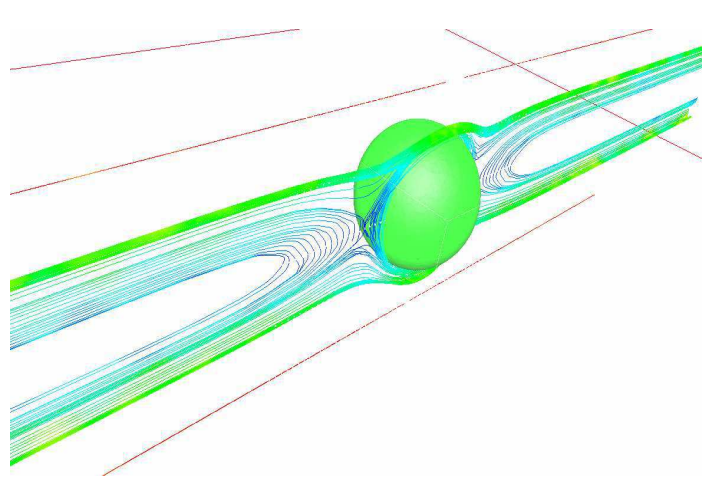

(b) $\mathrm{V}=\mathrm{d} / 4$

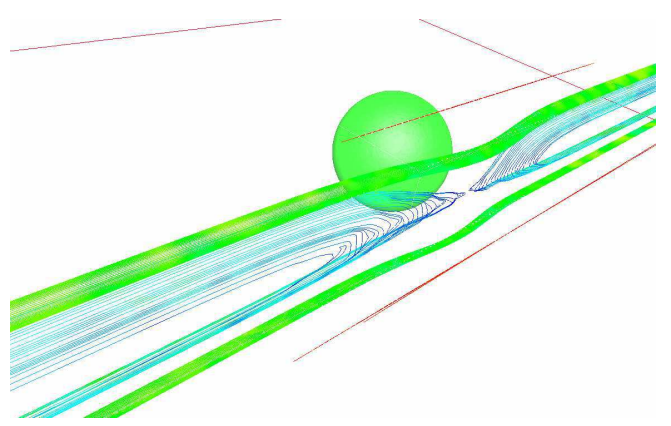

(d) $\mathrm{V}=\mathrm{d}$

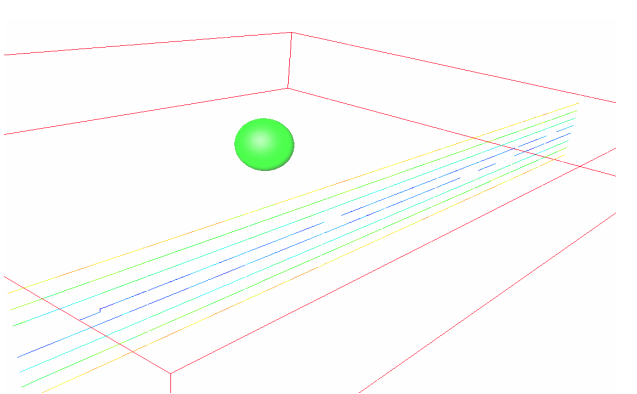

(f) $\mathrm{V}=4 \mathrm{~d}$

Figure 6 

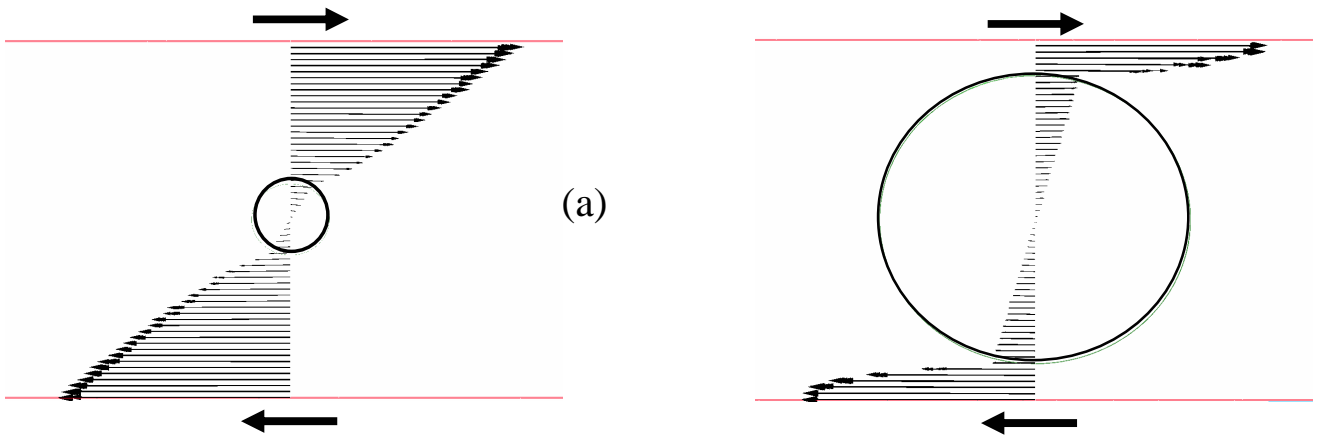

(b)

Figure 7 

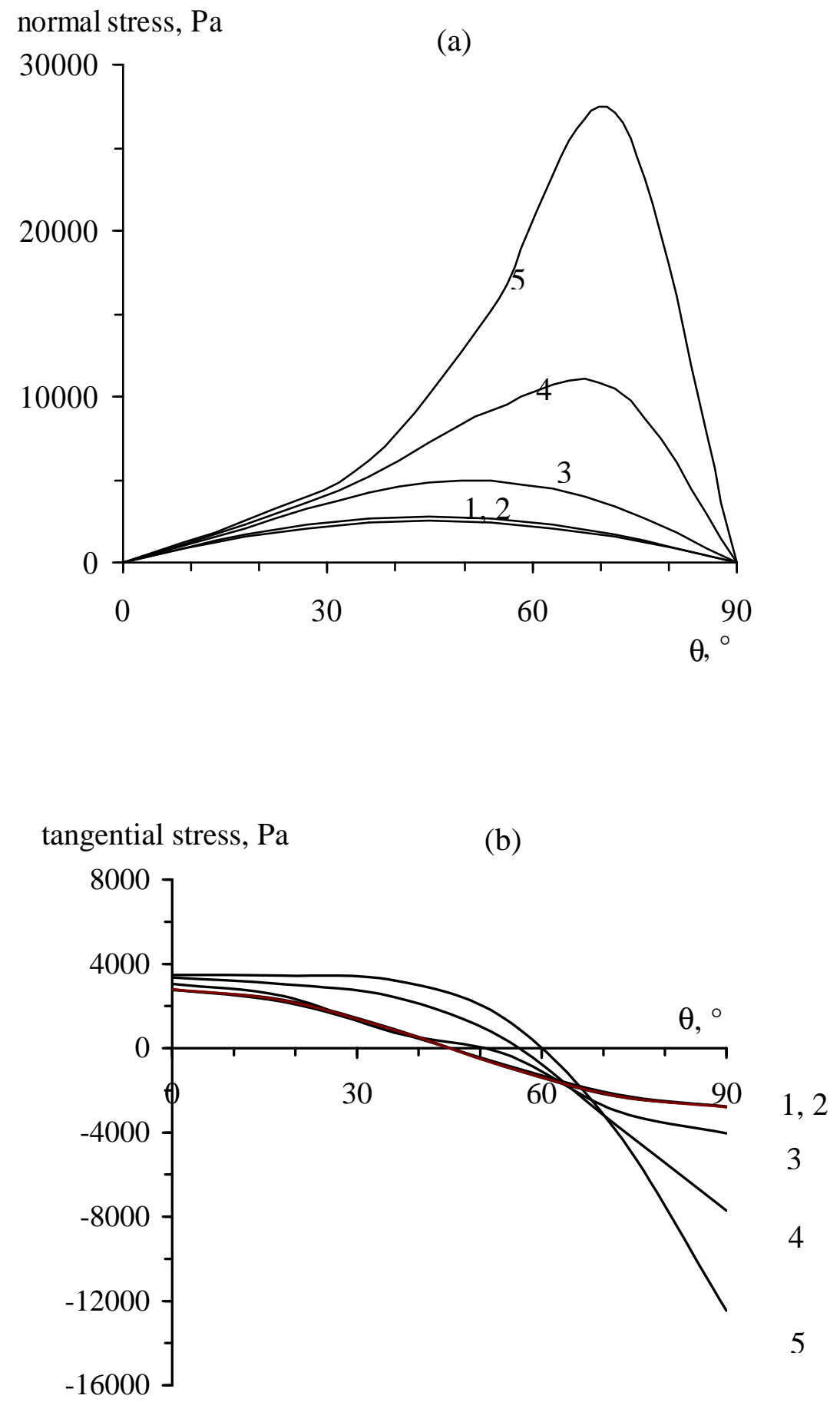

Figure 8 

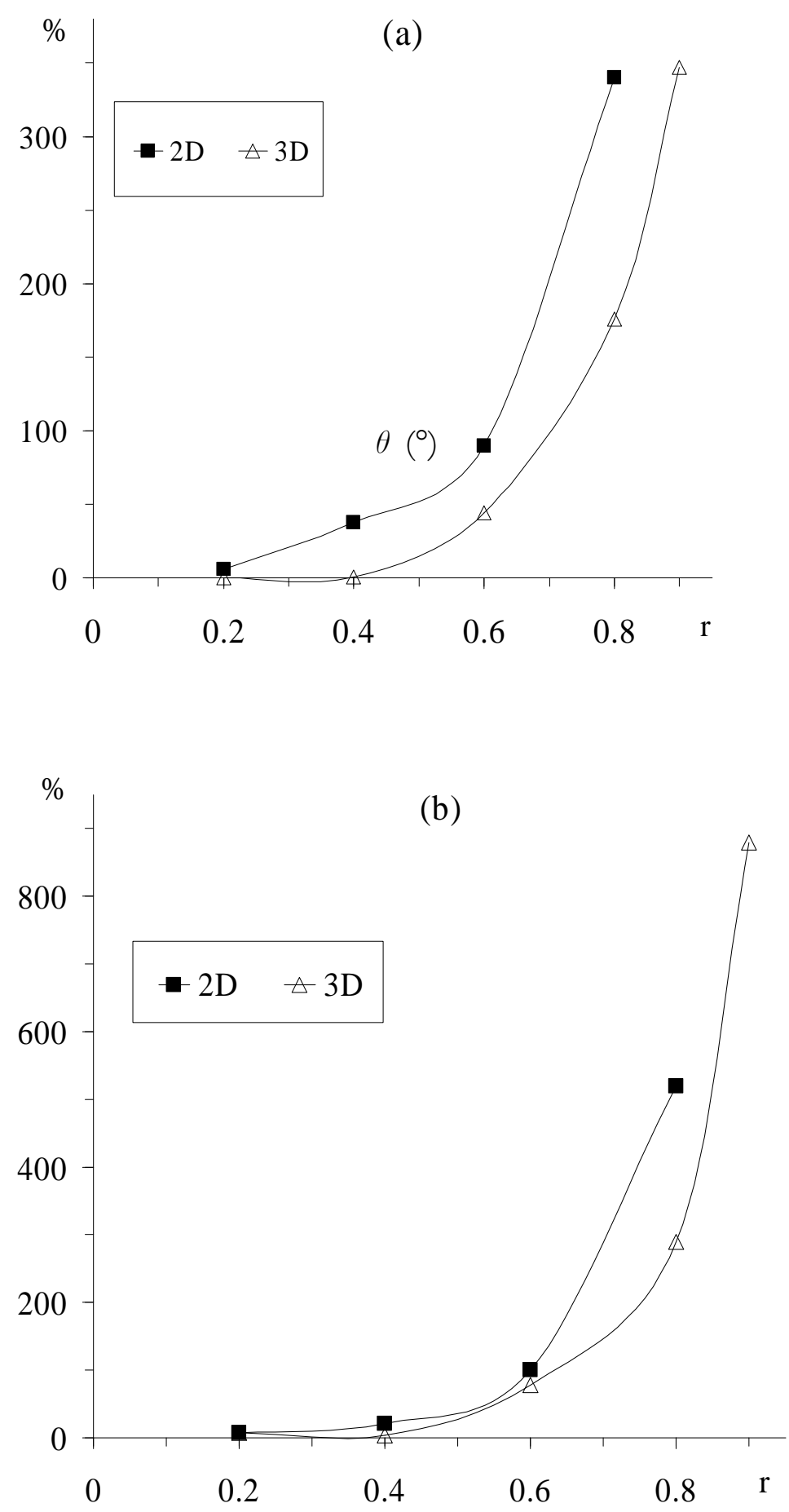

Figure 9 


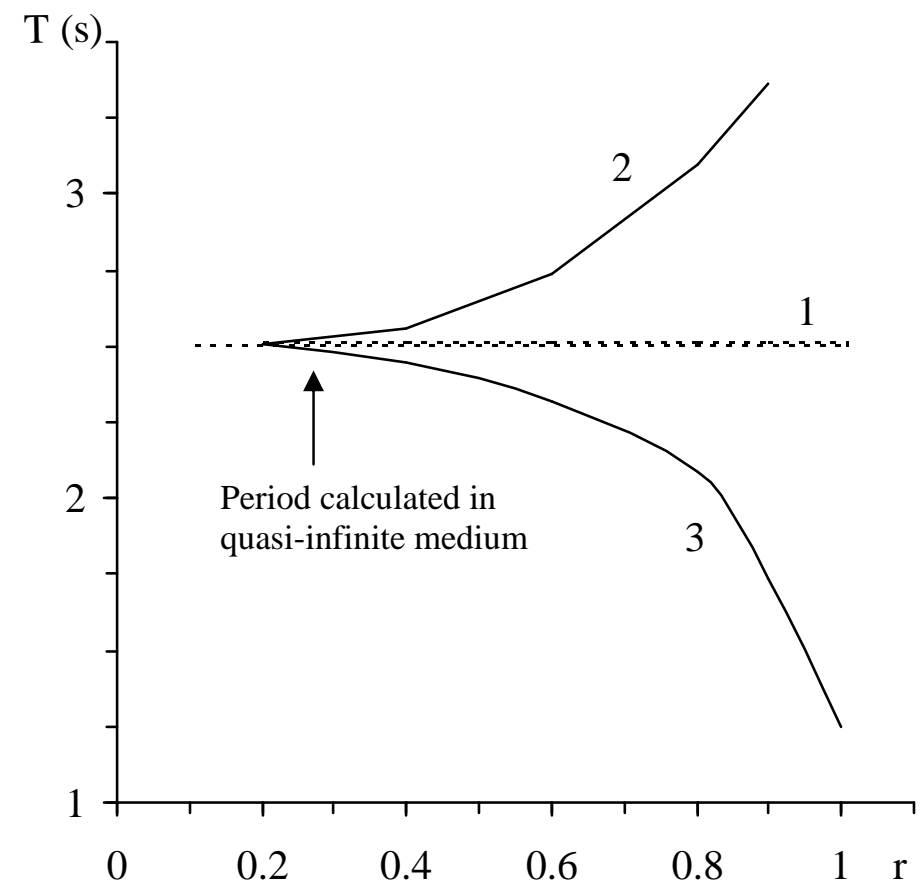

Figure 10 